\title{
Low-Level Laser Therapy versus Four Layers Compression Technique in the Treatment of Venous Ulcers
}

\author{
Medhat El-Laboudy, Ayman M. Samir*, Ahmad M. Tawfik, Waleed A. Sorour, Adel M. Tolba \\ Vascular Surgery Unit, Faculty of Medicine, Zagazig University, Zagazig, Egypt \\ Email: aymensamir@gmail.com
}

Received 18 July 2014; revised 15 August 2014; accepted 10 September 2014

Copyright (C) 2014 by authors and Scientific Research Publishing Inc.

This work is licensed under the Creative Commons Attribution International License (CC BY). http://creativecommons.org/licenses/by/4.0/

c) (7) Open Access

\section{Abstract}

Objective: The aim of the study is to evaluate the effect of low-level laser therapy in comparison to compression bandage therapy in the treatment of venous ulcers. Subjects \& Methods: This study was carried out in the period from March 2013 to March 2014. The study included 40 adult patients with a diagnosis of venous ulcer classified as $\mathrm{C} 6$ according to the clinical classification of CEAP classification. The patients were divided into 2 groups: the first one included 20 patients treated by low-level laser therapy at the Rheumatology and Rehabilitation department, and the second group included 20 patients in whom we use four layers compression bandaging at the vascular surgery department. Results: 32 ulcers were treated in group I and 35 ulcers were treated in group II. The patients of group I were 7 (35\%) females and $13(65 \%)$ males, their age range were (24 - 56) years. The patients of group II were $11(55 \%)$ males and $9(45 \%)$ females; their age ranges were $\left(32\right.$ - 50) years. Measurements of the ulcers size by $\left(\mathrm{cm}^{2}\right)$ were taken at the beginning of therapy, at 1 month, at 2 months and at 3 months later on. The calculation of the area of the ulcer was done by using the graph papers to document the ulcer's perpendicular linear dimensions. According to the size of the ulcer, some ulcers heal within 1 month $(15.6 \%)$ ulcers in group I, and (28.5\%) in group II. Some ulcers heal within 2 months $(28 \%)$ in group I, and $(37 \%)$ in group II. The remaining ulcers heal within 3 months or more which are (56\%) ulcers in group I, and $(34.2 \%)$ ulcers in group II. The recurrence rate of chronic venous leg ulcer in compression bandage technique used in group II was the least one as the total number of recurrent cases were $5 \mathbf{2 5 \%})$, followed by laser therapy used in group I. Conclusion: There was no significant efficacy of the low-level laser therapy over the four layers compression technique in the management of chronic venous ulcers.

\section{Keywords}

Venous Ulcer, Low-Level Laser, Four Layers Compression

\footnotetext{
*Corresponding author.

How to cite this paper: El-Laboudy, M., et al. (2014) Low-Level Laser Therapy versus Four Layers Compression Technique in the Treatment of Venous Ulcers. Surgical Science, 5, 403-410. http://dx.doi.org/10.4236/ss.2014.59065
} 


\section{Introduction}

The Chronic Venous Insufficiency (CVI) of the extremities is a progressive disease, and the most severe form of the disease is class 6 (according to the CEAP classification system), where active ulceration is formed. The venous ulcerations are located most often above the medial malleolus, are extremely hard to heal and are with distinct pain syndrome [1].

When the disease is not adequately treated, the patients may obtain various degrees of disability. The complex approach combining medicament and local treatment, limb elevation and elastic compression is the only method that has proven its effect in solving the problem because the presence of an active ulcer is a contra-indication against surgical intervention [2].

Below the knee, graduated compression from toe (highest) to knee (lowest), in the form of bandaging or stockings, is viewed as a key component of treatment when venous leg ulceration occurs in the absence of relevant arterial disease [3].

The impact of compression therapy on leg ulcer healing over the past 20 years has been quite significant, and cannot be ignored. The reality in clinical practice is that real improvements in outcome have only been seen in more recent years due to the technological advances in compression therapy, a greater understanding of the physiological mechanisms of compression and also a concerted effort to introduce compression into clinical practice in many parts of the world [4].

To many clinicians and scientists, the idea that low-level laser light can be therapeutic enough to relieve pain and promote tissue repair in collagenous tissues seems preposterous. Yet, some reports indicated that the lasers with $<500$ milliwatts ( $\mathrm{mW}$ ) average power, promote the repair processes of skin, ligaments, tendons, bone, and cartilage in experimental animals, as well as wounds and ulcers of a wide range of etiologies in humans [5].

A majority of animal experiments suggest that low-intensity lasers enhance wound healing by promoting cell proliferation, accelerating collagen synthesis and promoting the formation of granulation tissue, fostering the formation of type I and type III procollagen specific pools of mRNA, 58 increasing ATP synthesis within the mitochondria, activating lymphocytes, and increasing their ability to bind pathogens [6].

The availability of other studies that suggest the contrary, that is, the low-intensity lasers and other monochromatic light sources are not effective in promoting tissue repair, further complicates the matter, creating the present scenario in which low-intensity lasers are viewed with doubt [7].

The aim of the study is to evaluate the effect of low-level laser therapy in comparison to compression bandage therapy in the treatment of venous ulcers.

\section{Subjects and Methods}

\subsection{Study Design}

This study is a prospective non randomized clinical trial that was carried out on patients with venous ulcers. They were collected from the inpatient and outpatient clinic of vascular surgery unit in Zagazig University Hospitals.

Informed consent was taken from the patients, after receiving adequate information about the study (the characters of the study, benefits and possible side effects).

\subsection{Subjects}

This study was carried out in the period from March 2013 to March 2014.

The study included 40 adult patients of both sexes with a diagnosis venous ulcer classified as C6 according to the clinical classification of CEAP classification.

\section{Inclusion Criteria}

Any age, sex, one or more venous ulcers larger than $1 \mathrm{~cm}^{2}$ in area, no evidence of arterial disease of the legs (ankle brachial pressure index: 0.8), and patient not currently receiving any other form of treatment or dressing for the ulcer and ability to give written consent.

\section{Exclusion Criteria}

We excluded the patients with ulcers of infectious, pressure or postoperative origin, also, patients with uncon- 
trolled diabetes and diabetic sensory neuropathy, cellulitis, vasculitis or collagen vascular disease and any concomitant illnesses. Patients taking any medication that may affect wound healing, including corticosteroids and chemotherapy and patients who had active or suspected carcinoma were also excluded.

The calculation of the area of the ulcer was done by using the graph papers to document the ulcer's perpendicular linear dimensions (typically in centimeters using graph paper); the maximum distance is length and perpendicular distance is width. The progress of healing was measured every month and records the percent of healing.

The patients were classified in to 2 different groups of treatment.

\subsection{Group I}

It includes 20 patients treated by low-level laser therapy ( 7 females and 13 males). The procedure was carried out at the Rheumatology and Rehabilitation department.

\subsection{Group II}

It includes 20 patients in whom we use four layers compression bandaging ( 9 females and 11 males). The procedure was carried out at the vascular surgery department.

\section{Methods}

1) All patients subjected to a clinical sheet to collect data for clinical evaluation.

2) All patients were divided into two groups

3) All patients were subjected to the investigations:

- X-ray of leg and feet.

- Doppler ultrasound of both legs

Then For each group:

\subsection{Group I}

Twenty patients all subjected to low-level laser therapy to the ulcer. The infrared laser was applied by scanning method to the ulcer. We used Helium Neon Laser (gas mixture in pressurized tube), wave length $632.8 \mathrm{~nm}$, power output is $1.0-25 \mathrm{mw}$ and depth of $6-10 \mathrm{~mm}$. The treatment was applied for 3 months, 3 setting per week, with follow up every month to detect the improvement by measuring the diameter of the ulcer and detect the healing progression.

The patient lies comfortably on the bed, and an eye goggle is given to the patient to protect the eyes from the hazard of the low level laser therapy.

\subsection{Group II}

Twenty patients treated by compression therapy.

\section{Compression (bandaging) technique:}

Multi-layer graduated high compression elastic system (four layer bandage) was used in our study.

The so-called four-layer band age comprises 1) a padding bandage (orthopedic wool) to redistribution pressure from bony prominences and to help provide even distribution of pressure under the compression bandages where the shape of the leg may militate against even graduated pressure; 2) a crepe inelastic bandage (to create a base for the compression); and 3) and 4) two mild to moderate compression elastic bandages.

(1) Achieving effective pressure:

This four layer bandage is an example of a multi-component elastic system and is designed to apply a sustained sub-bandage pressure of $35-40 \mathrm{mmHg}$ at the ankle for patients with an ankle circumference of 18 - 25 $\mathrm{cm}$ in order to reverse chronic venous hypertension, this is achieved through:-

Laplace's law: theoretical pressures [5].

\section{Sub bandage pressure $P$ is dependent on:}

$$
\mathrm{P}=\frac{\mathrm{T} \times \mathrm{N} \times 462}{\mathrm{C} \times \mathrm{W}}
$$


$\mathbf{N}=$ Number of layers of bandage-more layers, the greater the pressure.

$\mathbf{T}=$ Tension in the bandage- - the greater the force applied, the greater the pressure

$\mathbf{C}=$ Limb circumference-the smaller the circumference, the greater the pressure.

$\mathbf{W}=$ Width of bandage - the narrower the bandage, the greater the pressure.

462 = The accepted constant.

La Place's Law can be used to predict the level of pressure that will be exerted upon a limb by a given bandage. The pressure generated by a bandage immediately following application is determined by tension in the fabric, number of layers applied and is inversely proportional to the circumference of the limb.

Therefore: Limb circumference affects the sub-bandage pressure as the thinner the leg, the more pressure will be exerted. And the more layers/overlaps are applied, the greater pressure.

(2) Application of bandaging:

a) Bandaging can be applied by a spiral technique with $50 \%$ overlapping and run from the base of the toes to just below the tibial plateau to be changed every week to avoid decrease of the applied pressure.

b) By starting at the base of the toes there is less chance of the bandage trapping interstitial fluid produced by normal systemic hydrostatic and osmotic pressure. In short, if the bandages started further up the foot or at the ankle there would be considerable swelling of the toes and forefoot. Application of the bandages up to the knee, just below the tibial plateau, ensures that the calf muscles are assisted in their pumping action, reducing superficial capillary and venous hydrostatic pressure by increasing the velocity of venous blood returning to the heart.

\subsection{Prevention of Recurrence}

By using elastic stocking and surgery, in which we did stripping of the long or the short saphenous veins with ligation of incompetent perforators, after complete healing of the ulcer.

The results from group I and group II was compared.

\section{The main variables for follow-up were the following:}

a) Measurement of the area of the lesions under aseptic conditions,

At the beginning: 1, 2, 3 months, by using a graph paper.

b) Qualitative clinical evaluation of the lesions.

- By physician, by inspection of the ulcer as regarding: color, inflammatory signs, infectious signs ,wound care, wound response and the recurrence.

- By the patient, by using the visual analogue scale.

A good result was considered when there was decrease in the diameters of the ulcer.

\subsection{Statistical Analysis}

Data entered, checked and analyzed by SPSS 10 software package. Quantitative data are summarized by mean and standard deviation for parameteric data. Non-parametric data are presented by median.

$\mathrm{P}$ value $<0.05$ was considered statistically significant, and $\mathrm{P}$ value $<0.001$ was considered highly significant. Differences between the groups in patients demographic were analyzed using one-way ANOVA (F test) and Fisher exact test.

\section{Results}

32 ulcers were treated in 20 patients in group I, which are distributed as follow: 6 patients have single ulcer in one leg, 4 patients have single ulcer in both legs, 5 patients have 2 ulcers in one leg and 2 patients have 2 ulcers in both legs.

35 ulcers were treated in 20 patients in group II, which are distributed as follow: 9 patients have single ulcer in one leg, 5 patients have single ulcer in both legs, 4 patients have 2 ulcers in one leg and 2 patients have 2 ulcers in both legs.

The patients of group I were 7 (35\%) females and 13 (65\%) males, their age range were (24 - 56) years. The cause of the ulcers is primary varicose veins in 6 patients and previous deep venous thrombosis (DVT) in 14 patients.

The patients of group II were 11 (55\%) males and 9 (45\%) females, their age range were (32 - 50) years. The cause of the ulcers is primary varicose veins in 8 patients and previous deep venous thrombosis (DVT) in 12 patients (Table 1). 
Table 1. Demographic criteria of the studied groups.

\begin{tabular}{ccccc}
\hline & Group I & Group II & $\mathbf{X}^{2}$ & P \\
\hline Sex: & & & & 0.45 \\
Male & $13(65 \%)$ & $11(55 \%)$ & 1.62 & \\
Female & $7(35 \%)$ & $9(45 \%)$ & & 0.43 \\
Cause of ulcer: & & & 1.68 & 0.89 \\
Incomp. Perforators & $1(5 \%)$ & $2(10 \%)$ & 0.42 & 0.93 \\
Incomp. Saph. Trunk & $2(10 \%)$ & $2(10 \%)$ & 0.15 & 0.74 \\
Incomp. Perf. \& Saph. Trunk & $3(15 \%)$ & $4(20 \%)$ & 0.60 & \\
Previous DVT & $14(70 \%)$ & $12(60 \%)$ & & \\
\hline
\end{tabular}

67 chronic venous leg ulcers in 40 patients were treated in our study, the cause of these venous ulcers are either 1ry varicose veins which are 6 (30\%) patients in group I, and 8 (40\%) patients in group II, or deep venous thrombosis (DVT) which are 14 (70\%) patients in group I, and 12 (60\%) patients in group II (Table 2).

Measurement of the ulcers size by $\left(\mathrm{cm}^{2}\right)$ was taken at the beginning of therapy, at 1month, at 2 months and at 3 months later on. The calculation of the area of the ulcer was done by using the graph papers to document the ulcer's perpendicular linear dimensions (typically in centimeters using graph paper); the maximum distance is length and perpendicular distance is width. The progress of healing was measured every month and records the percent of healing.

According to these measures we differentiate the ulcers into 3 categories. The $1^{\text {st }}$ category are ulcers whose size $<2 \mathrm{~cm}^{2}$ and this category include 7 ulcers in group I and 7 ulcers in group II. The $2^{\text {nd }}$ category is ulcers whose size 2 - $4 \mathrm{~cm}^{2}$ and this category include 16 ulcers in group I and 18 ulcers in group II. The $3^{\text {rd }}$ category are ulcers whose size $>4 \mathrm{~cm}^{2}$ and this category include 9 ulcers in group I and 10 ulcers in group II.

According to the size of the ulcer, some ulcers heal within 1 month which is $5(15.6 \%)$ ulcers in group I, and $10(28.5 \%)$ ulcers in group II. Some ulcers heal within 2 months which are $9(28 \%)$ ulcers in group I, and 13 (37\%) ulcers in group II. The remaining ulcers heal within 3 months or more which are 18 (56\%) ulcers in group I, and12 (34.2\%) ulcers in group II. Thus the percentage of regression of the total surface area are denoting that compression bandage technique used in group II is the most efficient in healing of chronic venous leg ulcer followed by laser therapy ( $\mathrm{P}=0.04$ at the end of the first month and $\mathrm{P}=0.03$ at the end of the third month) (Table 3).

In our study the patients were followed up for 1 year for detection of the recurrence, the patients were followed up at 3, 6 and 9 months after complete healing. After 3 months follow up there is recurrence in 2 patients (10\%) in group I and 1 patient (5\%) in group II while after 6 months follow up there is recurrence in group I, in 3 patients (15\%) and 2 patients (10\%) in group II. But after 9 months follow up, there is recurrence in 2 patients (10\%) in group I and 2 patients (10\%) in group II. Thus the recurrence rate of chronic venous leg ulcer in compression bandage technique used in group II is the least one as the total number of recurrent cases were 5 (25\%), followed by laser therapy used in group I as the total number of recurrent cases were 7 (35\%) (P = 0.85 nonsignificant).

\section{Discussion}

Our study showed that, some ulcers healed within 1 month which is 5 (15.6\%) ulcers in group I, and 10 (28.5\%) ulcers in group II, some ulcers healed within 2 months which are 9 (28\%) ulcers in group I, and 13 (37\%) ulcers in group II, the remaining ulcers healed within 3 months or more which are 18 (56\%) ulcers in group I, and 12 (34.2\%) ulcers in group II. Thus the percentage of regression of the total surface area are denoting that compression bandage technique used in group II is the most efficient in healing of chronic venous leg ulcer followed by laser therapy ( $\mathrm{P}=0.04$ at the end of the first month and $\mathrm{P}=0.03$ at the end of the third month).

The primary outcome measures were the mean time to heal, base line median ulcer duration was 3.8 months and base line median size was $3.7 \mathrm{~cm}^{2}$. The average reduction in total ulcer surface area was $1.2 \mathrm{~cm}^{2}$ per month. In these patients $90 \%$ of the wounds healed after 3 months, the mean healing time was 2 months, the healing was significant at the end of the first and third months more with compression therapy in group II followed by 
Table 2. Criteria of ulcer healing.

\begin{tabular}{|c|c|c|c|c|}
\hline & Group I & Group II & $\mathrm{X}^{2}$ & $\mathbf{P}$ \\
\hline \multicolumn{5}{|c|}{ Size of ulcer $\left(\mathrm{cm}^{2}\right)$ : } \\
\hline$<2 \mathrm{~cm}$ & $7(21.8 \%)$ & 7 (20\%) & 0.75 & 0.93 \\
\hline $2-4 \mathrm{~cm}$ & $16(50 \%)$ & $18(51.5 \%)$ & 3.73 & 0.15 \\
\hline$>4 \mathrm{~cm}$ & $9(28.1 \%)$ & 10 (28.5\%) & 0.40 & 0.82 \\
\hline \multicolumn{5}{|c|}{ Duration of ulcer healing: } \\
\hline $1^{\text {st }}$ month & $5(15.6 \%)$ & $10(28.5 \%)$ & 6.19 & $0.04^{*}$ \\
\hline $2^{\text {nd }}$ month & $9(28.4 \%)$ & $13(37 \%)$ & 1.54 & 0.46 \\
\hline $3^{\text {rd }}$ month & 18 (56\%) & $12(34.5 \%)$ & 7.2 & $0.03^{*}$ \\
\hline
\end{tabular}

Table 3. Qualitative clinical evaluation of the patient.

\begin{tabular}{|c|c|c|c|c|}
\hline & Group I & Group II & $\mathrm{X}^{2}$ & $\mathbf{P}$ \\
\hline \multicolumn{5}{|l|}{ Inflammatory signs: } \\
\hline Preprocedure & 18 (90\%) & 18 (90\%) & 0.44 & 0.83 \\
\hline \multicolumn{5}{|l|}{ During procedure } \\
\hline $1^{\text {st }}$ month & $12(60 \%)$ & $16(80 \%)$ & 3.73 & 0.15 \\
\hline $2^{\text {nd }}$ month & $6(30 \%)$ & $9(45 \%)$ & 1.78 & 0.41 \\
\hline $3^{\text {rd }}$ month & $2(10 \%)$ & $2(10 \%)$ & 5.57 & $0.06^{*}$ \\
\hline \multicolumn{5}{|l|}{ Infective signs: } \\
\hline Preprocedure & 15 (75\%) & $14(70 \%)$ & 0.48 & 0.49 \\
\hline \multicolumn{5}{|l|}{ During procedure } \\
\hline $1^{\text {st }}$ month & $11(55 \%)$ & $10(50 \%)$ & 0.82 & 0.49 \\
\hline $2^{\text {nd }}$ month & $6(30 \%)$ & $4(20 \%)$ & 0.50 & 1.37 \\
\hline $3^{\text {rd }}$ month & $2(10 \%)$ & $1(5 \%)$ & 0.77 & 0.54 \\
\hline \multicolumn{5}{|l|}{ Decrease of pain: } \\
\hline $1^{\text {st }}$ month & $11(55 \%)$ & $15(75 \%)$ & 3.33 & 0.19 \\
\hline $2^{\text {nd }}$ month & $5(25 \%)$ & $8(40 \%)$ & 6.85 & $0.04^{*}$ \\
\hline $3^{\text {rd }}$ month & $1(5 \%)$ & $1(5 \%)$ & 9.41 & $0.07^{*}$ \\
\hline \multicolumn{5}{|l|}{ Recurrence: } \\
\hline 3 months follow up & $2(10 \%)$ & $1(5 \%)$ & 2.26 & 0.32 \\
\hline 6 months follow up & 3 (15\%) & $2(10 \%)$ & 0.32 & 0.85 \\
\hline 9 months follow up & $2(10 \%)$ & $2(10 \%)$ & 0.32 & 0.85 \\
\hline Total No. & 7 (35\%) & $5(25 \%)$ & 1.76 & 0.42 \\
\hline
\end{tabular}

low level laser therapy in group I.

This is supported by a study done by Taradaj et al., who conducted a clinical trial with 40 patients with venous leg ulcers subjected to four layers compression bandages were compared with 2-layer short-stretch bandaging. For patients with isolated superficial reflux, the healing rates at 2 months were 10/22 healed (45.45\%) in group $\mathrm{A}$ and $4 / 22$ healed $(18.18 \%)$ in group $\mathrm{B}(\mathrm{P}=0.01)$. For patients with superficial plus deep reflux, the healing rates were $5 / 18$ healed (27.77\%) in group A and only $1 / 18$ healed $(5.55 \%)$ in group $B(P=0.002)$. Baseline median ulcer duration was 7.5 months and the baseline median size was $4.3 \mathrm{~cm}^{2}$. The average reduction in total ulcer surface was $2.9 \mathrm{~cm}^{2}$ per month. In these patients, $90.4 \%$ wounds healed after 12 months; the mean healing time was 3 months [8] [9].

In a study done by Jakub Taradaj et al., concludes that, the hemodynamic effect is one of the most significant biophysical mechanisms of healing after clinically efficient compression therapy. Hemodynamic reactions are 
not basic mechanisms of high voltage stimulation and ultrasound therapy during the healing of venous leg ulcers. Computed thermography is a simple and useful tool to measure hemodynamic effects in wound healing. They reported that low-level laser therapy can accelerate wound healing; these findings are supported by in vitro examinations confirming that low-level laser irradiation significantly increases cell proliferation and collagen deposition [10].

Milic et al., conducted a clinical trial to compare three different methods of compression in the treatment of venous ulcers. The healing rate during the 26-week treatment period was 13/42 (25\%) in group A; 31/46 (67.4\%) in group B; and 32/43 (74.4\%) in group C [11].

Brizzio et al., compared the proportion and rate of healing, pain, and quality of life between using Sigvaris prototype medical compression stockings (MCS), providing 15 - $25 \mathrm{mmHg}$ at the ankle and four layers compression bandaging applying 35 - $40 \mathrm{mmHg}$ at the ankle bandages. Healing within 90 days was observed in 36\% of patients using MCS and in 48\% using four layers compression bandages $(\mathrm{P}=0.35)$. Healing within 180 days was documented in 50\% of MCS patients and in 67\% with four layers compression bandages $(\mathrm{P}=0.21)$ [12].

A study done by Legan et al., concludes that Low intensity laser therapy, using single source irradiation, would seem to be an effective treatment for patients suffering venous ulceration. Treatments were carried out using single source irradiation (830 nm; $9 \mathrm{~J} / \mathrm{cm}^{2}$, CB Medico, Copenhagen, Denmark) in conjunction with dry dressings during each visit. Assessment of wound surface area, wound appearance, and current pain were completed by an independent investigator. Planimetry and digitizing were completed for wound tracings and for photographs to quantify surface areas. The primary findings were changes in wound appearance, and a decrease in wound surface area (range 33.3\% - 46.3\%), dependent on the choice of measurement method. Treatment intervention produced a statistically significant reduction in wound area using the $\mathrm{C}$ statistic on digitizing data for photographs (at Phase one only; $\mathrm{Z}=2.412$; $\mathrm{P}<0.05$ ). Wound debridement emerged as an important procedure to be carried out prior to measuring wounds. Despite fluctuating pain levels recorded throughout the duration of the study, VAS scores showed a decrease of $15 \%$ at the end of the study. This hypoalgesic effect was, however, statistically significant (using the $C$ statistic) at Phase one only ( $\mathrm{Z}=2.554$; $\mathrm{P}<0.05$ ) [13].

In contrast, Flemming and Cullumconducted e metanalysis study on trials comparing low level laser therapy with sham laser; no laser; non-coherent light. They found 4 eligible trials; two RCTs compared laser therapy with sham, 1 with ultraviolet therapy and 1 with non-coherent, unpolarised red light. Neither of the two RCTs comparing laser with sham found a significant difference in healing rates; there was no significant of laser evident when the trials were pooled. A three-arm study compared: laser therapy alone; laser therapy plus infrared light; non-coherent, unpolarised red light. Significantly more ulcers completely healed in the group receiving a combination of laser and infrared light compared with non-coherent, unpolarised red light. A fourth trial compared laser and ultraviolet light and found no difference. Finally, they found no evidence of any benefit associated with low level laser therapy on venous leg ulcer healing. One small study suggests that a combination of laser and infrared light may promote the healing of venous ulcers; however more research is needed [14].

Another study done by Cullum et al., found that compression is more effective in healing venous leg ulcers than is no compression, and multi-layered high compression is more effective than single-layer compression. High-compression hosiery was more effective than moderate compression in preventing ulcer recurrence. There is generally insufficient reliable evidence to draw conclusions about the contribution of laser therapy, therapeutic ultrasound, electrotherapy and electromagnetic therapy to chronic wound healing [15].

In our study, the recurrence rate of chronic venous leg ulcer in compression bandage technique, used in group II, is the least one; as the total number of recurrent cases were 5 (25\%), followed by laser therapy, used in group $\mathrm{I}$, as the total number of recurrent cases are 7 (35\%) ( $\mathrm{P}=0.85$ non-significant).

Lagatoll et al. conducted a randomized controlled trials performed on 136 patients subjected to four layers compression bandage with healed venous leg ulcers attending outpatients clinics for follow-up, there was recurrence in 32\% of patients with ulcers after twelve months of complete healing [16].

While in randomized controlled trials conducted by Wright et al., performed on 138 patients subjected to four layers compression bandage with healed venous leg ulcers attending outpatients clinic for follow up, there was recurrence in 34\% of patients after 18 months of complete healing [17].

Our analysis should be interpreted in the light of some potential limitations. First, the study sample was small, second, the follow up period was short and last, only two methods of venous ulcer treatment were compared. So, further studies with a larger sample, long term follow up and inclusion of other methods of ulcer treatment are necessary to confirm the findings of this study. 


\section{Conclusion}

This study did not show any superiority of low-level laser over compression bandaging in the treatment of venous ulcers. Our results did not show significant difference between both methods in the ulcer healing time, but the four layers compression showed shorter healing time in comparison to low-level laser therapy.

\section{References}

[1] Nicolaides, et al. (2008) Management of Chronic Venous Disorders of the Lower Limbs. A Guidlines According to Scientific Evidence. International Angiology, 1, 1-59.

[2] Pappas, P., et al. (2007) Pathophysiology of Chronic Venous Insufficiency. In: Bergan, J., Ed., The Vein Book, ElservierInc, London, 89-102.

[3] Hettrick, H. (2009) The Science of Compression Therapy for Chronic Venous Insufficiency Edema. The Journal of the American College of Certified Wound Specialists, 1, 20-24. http://dx.doi.org/10.1016/j.jcws.2008.10.002

[4] Cullum, N.A., et al. (2001) Compression for Venous Leg Ulcers (Cochrane Review). The Cochrane Library, Update Software Oxford.

[5] Basford, J.R., Hallman, H.O., Sheffield, C.G., et al. (1986) Comparison of Cold-Quartz Ultraviolet, Low-Energy Laser, and Occlusion in Wound Healing in a Swine Model. Archives of Physical Medicine and Rehabilitation, 67, 151-154. http://dx.doi.org/10.1016/0003-9993(86)90053-5

[6] Lubart, R., Friedmann, H., Sinyakov, M., et al. (1997) Changes in Calcium Transport in Mammalian Sperm Mitochondria and Plasma Membranes Caused by 780-nm Irradiation. Lasers in Surgery and Medicine, 21, 493-499. http://dx.doi.org/10.1002/(SICI)1096-9101(1997)21:5<493::AID-LSM12>3.0.CO;2-A

[7] Enwemeka, C.S. (1988) Laser Biostimulation of Healing Wounds: Specific Effects and Mechanism on Action. Journal of Orthopaedic \& Sports Physical Therapy, 9, 333-338. http://dx.doi.org/10.2519/jospt.1988.9.10.333

[8] Thomas, S. (2003) The Use of the Laplace Equation in the Calculation of Sub-Bandage Pressure. EWMA, 3, 21-23.

[9] Taradaj, J., Franek, A., Blaszczak, E., et al. (2012) Using Physical Modalities in the Treatment of Venous Leg Ulcers: A 14-Year Comparative Clinical Study. Wounds, 24, 215-226.

[10] Taradaj, J., Franek, A., Blaszczak, E., et al. (2012) Physical Therapy in the Treatment of Venous Leg Ulcers Disclosures. Wounds, 24, 138-145.

[11] Milic, D.J., Zivic, S.S., Bogdanovic, D.C., et al. (2010) The Influence of Different Sub-Bandage Pressure Values on Venous Leg Ulcers Healing When Treated with Compression Therapy. Journal of Vascular Surgery, 51, 655-661. http://dx.doi.org/10.1016/j.jvs.2009.10.042

[12] Brizzio, E., Amsler, F., Lun, B. and Blaettler, W. (2010) Comparison of Low-Strength Compression Stockings with Bandages for the Treatment of Recalcitrant Venous Ulcers. Journal of Vascular Surgery, 51, 410-416. http://dx.doi.org/10.1016/j.jvs.2009.08.048

[13] Lagan, K.M., McDonough, S.M., Clements, B.A. and Baxter, G.D. (2000) A Case Report of Low Intensity Laser Therapy (LILT) in the Management of Venous Ulceration: Potential Effects of Wound Debridement upon Efficacy. Journal of Clinical Laser Medicine and Surgery, 18, 15-22.

[14] Flemming, K. and Cullum, N. (2000) Laser Therapy for Venous Leg Ulcers. Cochrane Database of Systematic Reviews, 2, CD001182.

[15] Cullum, N., Nelson, E.A., Flemming, K. and Sheldon, T. (2001) Systematic Reviews of Wound Care Management: (5) beds; (6) Compression; (7) Laser Therapy, Therapeutic Ultrasound, Electrotherapy and Electromagnetic Therapy. Health Technology Assessment, 5, 1-221.

[16] Lagattolla, N.R.F., Burnand, K.G. and Eastham, D.A. (1995) Comparison of Perforating Vein Ligation, Stanozolol and Stockings in the Prevention of Recurrent Venous Ulceration. Phlebology, 10, 79-85.

[17] Wright, D., Franks, P., Blair, S., et al. (1991) Oxerutins in the Prevention of Recurrence in Chronic Venous Ulceration: Randomized Controlled Trial. British Journal of Surgery, 78, 1269-1270. http://dx.doi.org/10.1002/bjs.1800781039 
Scientific Research Publishing (SCIRP) is one of the largest Open Access journal publishers. It is currently publishing more than 200 open access, online, peer-reviewed journals covering a wide range of academic disciplines. SCIRP serves the worldwide academic communities and contributes to the progress and application of science with its publication.

Other selected journals from SCIRP are listed as below. Submit your manuscript to us via either submit@scirp.org or Online Submission Portal.
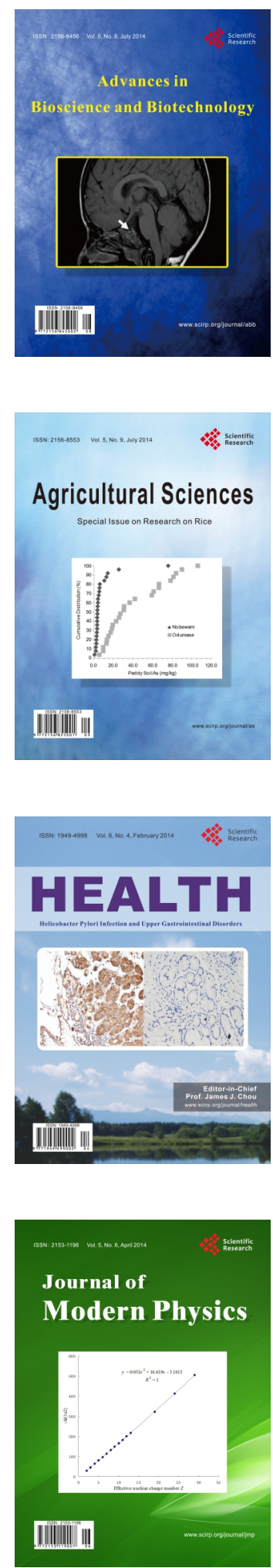
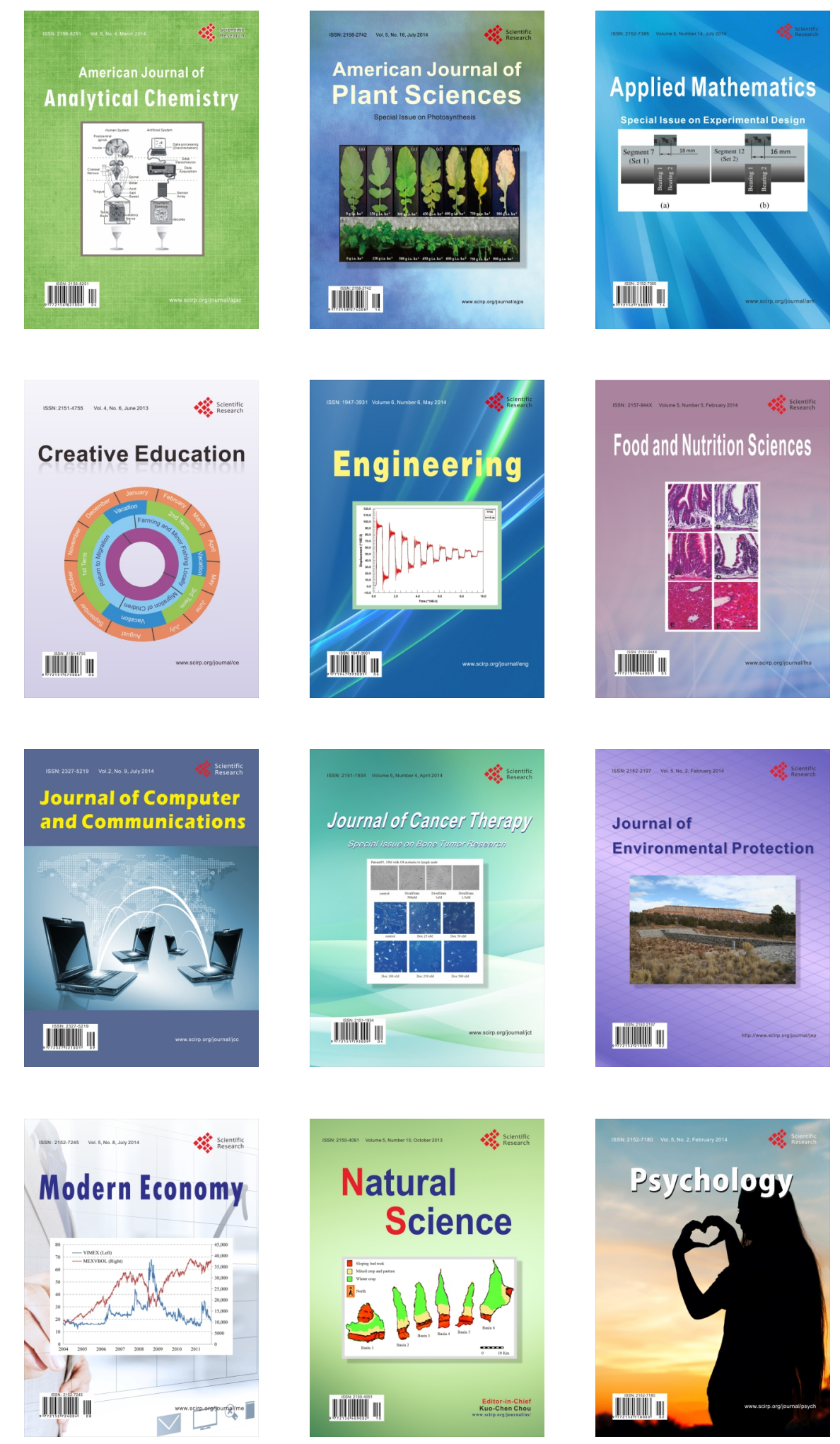